\title{
Survival and quality of life after extracorporeal life support for refractory cardiac arrest: A case series
}

\author{
Amedeo Anselmi, MD, PhD, ${ }^{\mathrm{a}, \mathrm{b}}$ Erwan Flécher, MD, PhD, ${ }^{\mathrm{a}, \mathrm{b}}$ Hervé Corbineau, MD,,${ }^{\mathrm{a}, \mathrm{b}}$ \\ Thierry Langanay, MD, ${ }^{\mathrm{a}, \mathrm{b}}$ Vincent Le Bouquin, MD, ${ }^{\mathrm{c}}$ Marc Bedossa, MD, ${ }^{\mathrm{d}}$ Alain Leguerrier, MD, \\ Jean-Philippe Verhoye, $\mathrm{MD}, \mathrm{PhD},{ }^{\mathrm{a}, \mathrm{b}}$ and Vito Giovanni Ruggieri, $\mathrm{MD}, \mathrm{PhD}^{\mathrm{a}, \mathrm{b}}$
}

\begin{abstract}
Objectives: Extracorporeal life support (ECLS) is an emerging option to treat selected patients with cardiac arrest refractory to cardiopulmonary resuscitation (CPR). Our primary objective was to determine the mortality at 30 days and at hospital discharge among adult patients receiving veno-arterial ECLS for refractory cardiac arrest. Our secondary objectives were to determine the 1-year survival and the health-related quality of life, and to examine factors associated with 30-day mortality.
\end{abstract}

Methods: In a retrospective, single-center investigation within a tertiary referral center, we analyzed the prospectively collected data of 49 patients rescued from refractory cardiac arrest through emergent implantation of ECLS (E-CPR) (18.1\% of our overall ECLS activity, 2005-2013), implanted in-hospital and during ongoing external cardiac massage in all cases. A prospective follow-up with administration of the Medical Outcomes Study 36-Item Short-Form Health Survey (SF-36) questionnaire was performed.

Results: The mean age was $47.6 \pm 1.6$ years; out-of-hospital cardiac arrest occurred in $12 \%$ of cases; average low-flow time was $47.2 \pm 33$ minutes; causes of cardiac arrest were heart disease $(61.2 \%)$, trauma $(14.3 \%)$, respiratory disease $(4.1 \%)$, sepsis $(2 \%)$, and miscellaneous (18.4\%). Primary objective: Rates of survival at E-CPR explantation and at 30 days were $42.9 \%$ and $36.7 \%$, respectively; brain death occurred in $24.5 \%$ of cases. Secondary objectives: Increased simplified acute physiology score; higher serum lactate levels and lower body temperature at the time of implantation were associated with 30-day mortality. Bridge to heart transplantation or implantation of a long-term ventricular assist device was performed in $8.2 \%$. No deaths occurred during the follow-up after discharge $(36.7 \%$ survival; average follow-up was $15.6 \pm 19.2$ months). The average Physical Component Summary and Mental Component Summary scores (SF-36 questionnaire) were, respectively, $45.2 \pm 6.8$ and $48.3 \pm 7.7$ among survivors.

Conclusions: Extracorporeal cardiopulmonary resuscitation is a viable treatment for selected patients with cardiac arrest refractory to CPR. In our series, approximately one third of rescued patients were alive at 6 months and presented quality-of-life scores comparable to those previously observed in patients treated with ECLS. (J Thorac Cardiovasc Surg 2015;150:947-54)

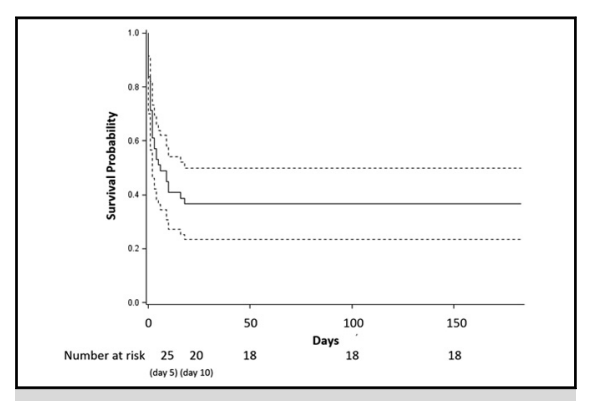

Survival curve for patients receiving extracor poreal cardiopulmonary resuscitation (follow-up: $15.6 \pm 19.2$ months).

\section{Central Message}

Extracorporeal cardiopulmonary resuscitation can achieve $36.7 \%$ survival at midterm in selected patients with cardiac arrest refractory to cardiopulmonary resuscitation.

\section{Perspective}

Selection of patients is key to achieving adequate results with E-CPR. Among survivors, quality of life at follow-up was comparable to that previously obtained after ECLS therapy. A multidisciplinary debate is needed to establish evidence-based protocols and justified use of ECLS. The diffusion of ECLS needs to take into account organizational, economic, ethical, and learning-curve issues.

See Editorial Commentary page 955

\footnotetext{
From the a Division of Thoracic, Cardiac and Vascular Surgery, Pontchaillou University Hospital; ${ }^{\mathrm{b}}$ INSERM (French National Institution for Healthcare and Medical Research), Unit 1099, University of Rennes 1; 'Division of Cardiac Anesthesia, Pontchaillou University Hospital; and ${ }^{\mathrm{d}}$ Division of Cardiology and Cardiac Intensive Care Unit, Pontchaillou University Hospital, Rennes, France.

Received for publication Dec 14, 2014; revisions received April 18, 2015; accepted for publication May 30, 2015; available ahead of print July 15, 2015.
}

\footnotetext{
Address for reprints: Amedeo Anselmi, MD, PhD, Division of Thoracic, Cardiac an Vascular Surgery, Rennes University Hospital, 2 rue Henri Le Guilloux, 35033 Rennes, France (E-mail: amedeo.anselmi@alice.it). $0022-5223 / \$ 36.00$

Copyright $@ 2015$ by The American Association for Thoracic Surgery http://dx.doi.org/10.1016/j.jtcvs.2015.05.070
} 


\section{Abbreviations and Acronyms \\ $\mathrm{CPR}=$ cardiopulmonary resuscitation \\ ECLS = extracorporeal life support \\ $\mathrm{ECMO}=$ extracorporeal membrane oxygenation \\ $\mathrm{E}-\mathrm{CPR}=$ extracorporeal cardiopulmonary resuscitation \\ IABP $=$ intra-aortic balloon pump \\ SF-36 = Medical Outcomes Study 36-Item Short- Form Health Survey (French version)}

Extracorporeal life support (ECLS) therapy is an option for severe cardiac or cardiopulmonary failure, and consists of an extracorporeal circulation circuit equipped with a rotary blood pump and a blood hollow-fiber oxygenator. Increasing evidence shows the usefulness of ECLS in improving the survival of selected patients who have cardiac arrest refractory to extracorporeal cardiopulmonary resuscitation (E-CPR). ${ }^{1}$ The survival at 30 days after ECLS implantation in such patients reportedly ${ }^{2-4}$ ranges between $24 \%$ and $36 \%$. Other series have reported survival rates up to $50 \% .^{5}$ These studies are difficult to compare with ours because they included patients who received ECLS implantation in cases other than with cardiopulmonary resuscitation (CPR), or in-hospital cardiac arrest. ${ }^{5}$

Little evidence is available regarding the clinical outcomes after hospital discharge of patients who survived both refractory cardiac arrest and failed CPR, owing to ECLS therapy. This population consists of critically ill patients who have high rates of severe in-hospital complications. Therefore, not only the vital status of these patients late after hospital discharge needs to be clarified, but also their quality of life. The latter information is lacking even in data from major multicenter registries, ${ }^{6}$ even though it may be important in clinical decision making and allocation of resources. Projected quality of life is cited in the recent European guidelines ${ }^{6}$ as one of the primary aspects to be considered in evaluating the indication for ECLS in acute heart failure. However, such judgment poses major difficulties among patients with refractory cardiogenic shock, owing to emergent presentation, frequent uncertainty of the extent of brain damage, and coexistence of comorbidities. The literature lacks specific information on this issue as well.

The purpose of the present investigation was 2-fold. The first objective was to clarify the mortality at 30 days, and at hospital discharge, in adult patients receiving veno-arterial ECLS for refractory cardiac arrest. The secondary objectives were to clarify the 1-year survival and perceived health-related quality of life in these patients, and to identify factors associated with 30-day mortality.

\section{METHODS \\ Selection of Patients and ECLS Implantation \\ Technique}

Between January 2005 and October 2013, a total of 270 patients received veno-arterial ECLS at our institution. Of these, $49(18.1 \%)$ were treated on an emergent basis for cardiac arrest refractory to conventional CPR. All the patients in the present study received ECLS implantation during ongoing CPR. We excluded patients who were given ECLS immediately after cardiotomy, owing to the impossibility of weaning them from cardiopulmonary bypass; in addition, patients receiving venovenous ECLS for primary lung dysfunction were excluded. No patient received E-CPR out-of-hospital; nevertheless, some patients who suffered cardiac arrest out-of-hospital and received E-CPR within our center were included $(\mathrm{N}=6 ; 12 \%)$. The cases satisfying the above criteria were retrospectively selected from our database and used for subsequent analyses.

The indications for E-CPR were established in compliance with current recommendations, ${ }^{6}$ by a multidisciplinary team that included at least 1 surgeon and 2 physicians. We adopted the algorithm proposed by Combes and colleagues. ${ }^{7}$ Briefly, E-CPR was contraindicated in cases of: no-flow time $>5$ minutes, or unwitnessed cardiac arrest; total expected duration of low-flow $>100$ minutes; end-expiratory $\mathrm{CO}_{2}$ concentration $<10 \mathrm{~mm}$ $\mathrm{Hg}$; and asystole on electrocardiogram.

In addition, the indication for E-CPR was discussed in relation to the patient's known comorbidities and life expectancy. Intoxication or hypothermia $\left(\leq 32^{\circ} \mathrm{C}\right)$ were elements supporting the indication for E-CPR during enrollment of the present series. The implanting team included 2 surgeons (senior and resident), a scrub nurse, and a perfusionist. All material is available on a dedicated trailer, allowing full autonomy for prompt deployment of the team within the hospital facilities.

Implantation of E-CPR was performed through a longitudinal skin incision at the right groin, and preparation of the anterior face of the common femoral artery and vein. The femoral vessels were neither encircled nor clamped. The percutaneous technique was never employed, to facilitate rapid institution of E-CPR and avoid the difficulties of monitoring pulses and backflow resulting from marginal hemodynamic conditions. Cannulation was performed using the Seldinger technique; arterial inflow was obtained through a 16 to $20 \mathrm{Fr}$ cannula; venous drainage was achieved with an 18 to $24 \mathrm{Fr}$ cannula (both Edwards Lifesciences, Irvine, Calif). In the event of failed cannulation owing to extensive arterial calcifications, or inability to adequately advance the guidewires, the left groin was explored. Distal perfusion of the lower limb was assured through the superficial femoral artery, using a 6 to 10 Fr cannula (Seldinger technique) connected to a side branch of the arterial inflow line.

During ECLS therapy, heparin was administered through a central venous line to maintain an activated clotting time of between 150 and 180 seconds; in the presence of hemorrhagic complications, the dose of heparin was adjusted, or the administration was temporarily withheld. Efficacy of ECLS therapy was monitored by periodic assessment of arterial and central venous pressures, urine output, systemic oxygen saturation, and venous lactate concentration, as reported in the current recommendations. ${ }^{8}$ Pump speed was adjusted to keep the cardiac index within the 2.2 to $2.81 \mathrm{~min}^{-1} \mathrm{~m}^{-2}$ range, and to obtain adequate cardiac unloading. Patients on ECLS were evaluated on a daily basis by a multidisciplinary team that included surgeons, cardiologists, and anesthesiologists. Transthoracic and/or transesophageal echocardiography were used for verification of cardiac decompression.

In the event of inadequate left ventricular unloading and/or the occurrence of pulmonary edema, adjustment of inotropic drugs (dobutamine) and/or intra-aortic balloon pump (IABP) therapy were adopted. A shift to central cannulation and surgical left ventricular unloading through the right upper pulmonary vein or main pulmonary artery was considered as a further measure during the earliest part of 
our experience. Later on, the positioning of a transaortic axial flow pump (Impella, Abiomed Inc, Aachen, Germany) was considered.

The oxygenator and circuit were replaced in the case of clot formation within the system, or major hemolysis. If IABP therapy was already in place at the time of E-CPR implantation, it was maintained until the end of ECLS. Therapeutic hypothermia was performed by at least 24 hours (target: $34^{\circ} \mathrm{C}$ ). Weaning from ECLS was considered when partial or complete recovery from cardiac dysfunction could be demonstrated on echocardiography, associated with the presence of a stable pulsatile blood pressure waveform, and mean blood pressure remaining $>60 \mathrm{~mm} \mathrm{Hg}$ during a weaning trial (progressive reduction of the ECLS flow until a minimum of 11 per minute), with administration of minimal- or low-dose inotropic agents.

\section{Definitions}

We defined refractory cardiac arrest as persisting beyond the 30th minute of CPR. Etiologies of cardiac arrest were categorized as follows: cardiac disease (further divided into acute myocardial infarction, cardiomyopathy, acute myocarditis, refractory arrhythmia, acute pulmonary embolism); respiratory disease; trauma (including hypothermia, suicide attempt, drug overdose); sepsis; and miscellaneous (cases that could not be categorized into the other groups). Vascular complications at the site of E-CPR implantation were: need for $\geq 1$ additional surgical procedure to revise hemostasis at the site of cannulation for ECLS, or to reconstruct/repair the involved vessels. Lower-limb ischemia was defined as the need for $\geq 1$ additional surgical procedure to relieve ischemia and/or treat compartmental syndrome.

Hemorrhagic complications of ECLS were defined as need for $\geq 1$ surgical revision for bleeding, at a site other than that of the cannulated vessels; and/or for transfusion of $\geq 4$ units of concentrated red blood cells to treat anemia resulting from bleeding. Complications of ECLS were grouped according to the Extracorporeal Life Support Organization registry definitions, as follows: ECLS circuit; central nervous system; cardiac; pulmonary; infectious; renal; gastrointestinal; and metabolic. ${ }^{5}$ Brain death of patients on ECLS had to be declared by consensus of $\geq 2$ intensive-care medicine specialists, according to the criteria established by French law (no brainstem reflexes; absence of spontaneous ventilation at hypercapnia test $\left[\mathrm{P}_{\mathrm{CO} 2}>60 \mathrm{~mm} \mathrm{Hg}\right]$; and 2 electroencephalography recordings taken within a 4-hour interval). Patients were evaluated using the simplified acute physiology score (version 2) at the time of E-CPR implantation, ${ }^{9}$ using the French Society of Anesthesia and Intensive Care (Paris, France) online calculator.

\section{Follow-up and Quality-of-Life Assessment}

A follow-up program for patients receiving ECLS at our institution was started in April 2013. Patients who were alive at the time of hospital discharge were contacted by phone, by research nurses experienced in the chronic management of patients receiving surgical treatment for heart failure. All patients could be contacted and interviewed directly. The inquiry included assessment of their functional and vital status, adverse events after discharge, and formal evaluation of quality of life among survivors (via administration of the French version of the Medical Outcomes Study 36-Item Short-Form Health Survey; SF-36). ${ }^{10-12}$ The questionnaire was administered according to recommendations, ${ }^{10}$ and provided a score for each of the following domains: Physical Activity, Role Limitation, Physical Pain, General Health, Vitality, Social Activity, Mental Health, Physical Component Summary, and Mental Component Summary. The SF-36 questionnaire has been previously employed in the evaluation of health-related quality of life in patients who survive critical illness because of $\mathrm{ECLS}^{13}$ or venovenous extracorporeal membrane oxygenation, ${ }^{14}$ and in patients with advanced heart failure. ${ }^{15-17}$ In addition, the SF-36 has been used to evaluate survivors of cardiac arrest who have not been treated by E-CPR. ${ }^{18}$ Data were entered into the Rennes ECLS Registry.

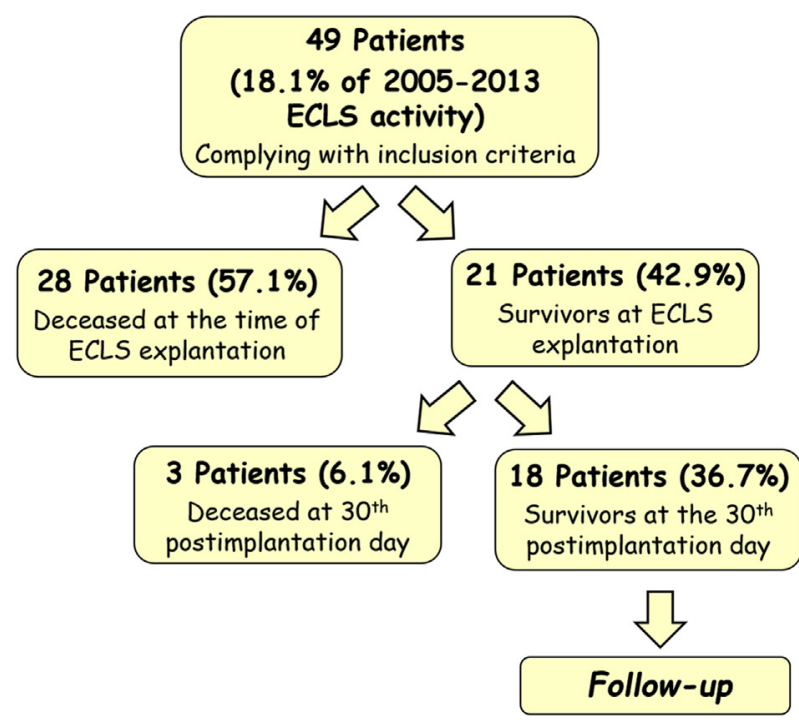

FIGURE 1. Study design. ECLS, Extracorporeal life support.

\section{Management of Data}

Since the beginning of the ECLS program at our institution, the clinical data pertaining to each patient receiving such treatment are prospectively entered in an electronic database. This information includes all pre- and post-implantation data, major biological and hemodynamic parameters, drug therapy, and in-hospital complications. The database is regularly checked by dedicated personnel for errors and omissions. The prospectively collected data can be retrospectively analyzed. Institutional review board approval was obtained. Our study is reported to the CLIN online database (Commission Nationale de l'Informatique et des Libertées, ie, the French National Commission for Informatics and Freedom), according to French law.

\section{Statistical Analysis}

Continuous data are presented as mean $\pm \mathrm{SD}$; categoric variables are reported as percentages. Intergroup comparison was performed using the 2 -tailed Student $t$ test for continuous data, and $\chi^{2}$ analysis for categoric data. All available baseline variables were used for intergroup comparison (approximately 40 variables). Survival analysis was conducted using the Kaplan-Meier method, and the corresponding survival curves were built. Analyses were performed using SAS software, version 9.33 for Windows (SAS Institute Inc, Cary, NC).

\section{RESULTS}

The study workflow is reported in Figure 1. Forty-nine patients received E-CPR (18.1\% of overall ECLS activity at our institution). The baseline features of the study population and the indications for E-CPR treatment are summarized in Table 1. Most frequently, the indication for E-CPR pertained to the cardiac disease group; among these, acute myocardial infarction was predominant $(47 \%$ of the 30 patients in this group). Two patients had baseline respiratory disease, which led to anoxic refractory cardiac arrest and required E-CPR. In these patients, circulatory and myocardial failure in conjunction with pulmonary failure made the use of venovenous extracorporeal 
TABLE 1. Characteristics of the study population

\begin{tabular}{|c|c|}
\hline Characteristic & Value \\
\hline Age (y) & $47.6 \pm 16$ \\
\hline Gender: male; female & $30 ; 19(61.2 ; 38.8)$ \\
\hline Body surface area $\left(\mathrm{m}^{2}\right)$ & $1.89 \pm 0.23$ \\
\hline \multicolumn{2}{|l|}{ Site of cardiac arrest } \\
\hline In-hospital & $43(88)$ \\
\hline Out-of-hospital & $6(12)$ \\
\hline \multicolumn{2}{|l|}{ Site of E-CPR implantation } \\
\hline Operative theater & $24(49)$ \\
\hline Intensive-care unit & $18(36.7)$ \\
\hline Catheterization room & $2(4.1)$ \\
\hline Other & $5(10.2)$ \\
\hline \multicolumn{2}{|l|}{ Indication for E-CPR } \\
\hline Cardiac disease & $30(61.2)$ \\
\hline Respiratory disease & $2(4.1)$ \\
\hline Trauma & $7(14.3)$ \\
\hline Sepsis & $1(2)$ \\
\hline Miscellaneous & $9(18.4)$ \\
\hline LVEF* $(\%)$ & $22.8 \pm 15.3$ \\
\hline No-flow time (min) & $1.1 \pm 2.9$ \\
\hline Low-flow time (min) & $47.2 \pm 33$ \\
\hline \multicolumn{2}{|l|}{ Inotropes $\dagger$} \\
\hline Dobutamine $>5 \mathrm{\gamma} / \mathrm{kg} / \mathrm{min}$ & $18(36.7)$ \\
\hline Adrenaline $>1 \mathrm{mg} / \mathrm{h}$ & $16(32.6)$ \\
\hline Noradrenaline $>1 \mathrm{mg} / \mathrm{h}$ & $25(51)$ \\
\hline $\mathrm{pH}$ & $7.2 \pm 0.3$ \\
\hline Serum lactates $(\mathrm{mmol} / \mathrm{L})$ & $11.7 \pm 6$ \\
\hline Serum creatinine $(\mu \mathrm{mol} / \mathrm{L})$ & $144 \pm 53$ \\
\hline SGOT (IU/L) & $637 \pm 961$ \\
\hline Troponin $\mathrm{T}$ (nanog/L) & $472 \pm 1188$ \\
\hline SAPS & $61.1 \pm 29.2$ \\
\hline \multicolumn{2}{|c|}{$\begin{array}{l}\text { Values are mean } \pm \mathrm{SD} \text {, or } \mathrm{n}(\%) . E-C P R \text {, Extracorporeal cardiopulmonary } \\
\text { resuscitation; } L V E F \text {, left ventricular ejection fraction; SGOT, serum glutamic } \\
\text { oxaloacetic transaminase; SAPS, simplified acute physiology score. *Any LVEF } \\
\text { measurement available on the day of E-CPR implantation, including LVEF } \\
\text { immediately after institution of support. †Catecholamines administered while patient } \\
\text { was on support, excluding those administered during cardiopulmonary resuscitation. }\end{array}$} \\
\hline
\end{tabular}

membrane oxygenation (ECMO) alone insufficient. Biological variables at the time of implantation (serum lactate concentration, $\mathrm{pH}$, serum creatinine, and markers of end-organ damage) highlight the critical general conditions of these patients. Average no-flow time was $47.2 \pm 33$ minutes. Average diameter of the arterial and venous cannulae was $19.1 \pm 1.5 \mathrm{Fr}$ and $20.3 \pm 2.6 \mathrm{Fr}$, respectively.

Twenty-one patients $(42.9 \%)$ were alive at the time of explantation of ECLS; among these, the average duration of support was $6.8 \pm 5.8$ days. The remaining patients $(57.1 \%)$ died after $2.8 \pm 4$ days after institution of E-CPR. Three patients died within the hospital after explantation of ECLS, and by the 30th day after implantation. Causes of death were: multiorgan failure in 14 cases $(28.6 \%$ in the overall population); brain death in 12 cases $(24.5 \%)$; and irrecoverable myocardial failure in 2 cases. No further patients died within the hospital after
TABLE 2. Survivors versus nonsurvivors at 30 days after implantation of E-CPR: univariate intergroup comparison of patient-related variables at the time of start of support

\begin{tabular}{lccc}
\hline \multicolumn{1}{c}{ Characteristic } & $\begin{array}{c}\text { Survivors } \\
(\mathbf{n}=\mathbf{1 8})\end{array}$ & $\begin{array}{c}\text { Nonsurvivors } \\
(\mathbf{n}=\mathbf{3 1})\end{array}$ & $\begin{array}{c}\boldsymbol{P} \\
\text { value }\end{array}$ \\
\hline Gender: male & $13(72.2)$ & $17(54.8)$ & .2 \\
Age $(\mathrm{y})$ & $44.9 \pm 17.3$ & $48.8 \pm 15.3$ & .2 \\
Site of implantation & & & \\
$\quad$ Operative theater & $11(61)$ & $13(41.9)$ & \\
$\quad$ Other & $7(39)$ & $18(58.1)$ & .19 \\
SAPS & $43.5 \pm 21.5$ & $70.3 \pm 28.8$ & .006 \\
Body temperature at & $36 \pm 1.9$ & $34.7 \pm 2.2$ & .02 \\
$\quad$ E-CPR implantation $\left({ }^{\circ} \mathrm{C}\right)$ & & & \\
Serum creatinine $(\mu \mathrm{mol} / \mathrm{L})$ & $139 \pm 53$ & $148 \pm 53$ & .5 \\
Serum lactates $(\mathrm{mmoL} / \mathrm{L})$ & $8.6 \pm 4.4$ & $12.8 \pm 6.6$ & .04 \\
pH & $7.28 \pm 0.3$ & $7.2 \pm 0.2$ & .4 \\
Low-flow time $(\mathrm{min})$ & $33.4 \pm 15.6$ & $52.9 \pm 36.6$ & .09 \\
No-flow time $(\min )$ & 0 & $1.6 \pm 3.3$ & .16 \\
\hline Values are mean $\pm \mathrm{SD}$, or $\mathrm{n}(\%) . E-C P R$, Extracorporeal cardiopulmonary \\
resuscitation; SAPS, simplified acute physiology score.
\end{tabular}

the 30th day after implantation. Thus, 18 patients ( $36.7 \%$ of the initial population) were discharged alive from the hospital (average hospital stay: $79.1 \pm 97.6$ days).

Thromboembolic and hemorrhagic complications were common under ECLS (59.2\% cumulative rate). Only one patient experienced an ischemic complication of the lower $\operatorname{limb}(2 \%)$. Transfusion with red blood cells, platelets units, and fresh frozen plasma units was required in $79.1 \%$, $54.2 \%$, and $61.2 \%$ of cases, respectively. Seven patients $(14.3 \%)$ were concomitantly treated by IABP; among these, 3 received IABP after E-CPR implantation, to facilitate unloading of the left ventricle. Two patients $(9.5 \%$ of survivors at explantation) were successfully bridged to heart transplantation, and 2 additional patients received an implantable left ventricular assist device.

At the time of ECLS explantation, a marked improvement of the biological parameters was evident: average $\mathrm{pH}$ was $7.4 \pm 0.1$; serum lactates concentration was $2.8 \pm 3.3 \mathrm{mmol} / \mathrm{L}$. Partial recovery of end-organ damage was disclosed (average serum glutamic oxaloacetic transaminase concentration: $82.3 \pm 78.5 \mathrm{IU} / \mathrm{L}$; serum creatinine: $116.8 \pm 93 \mu \mathrm{mol} / \mathrm{L})$. Cardiac function was partially recovered as well, the average left ventricular ejection fraction being $37 \% \pm 15 \%$. Brain death occurred in 12 cases $(24.5 \%$ of the entire population; $38.7 \%$ of deceased patients). The average duration of mechanical ventilation was $7.7 \pm 9.2$ days. Table 2 compares the major patient-related variables among survivors and nonsurvivors at the 30th day after implantation. A higher simplified acute physiology score, lower body temperature, and higher lactate level at the time of ECLS implantation were statistically associated with death before explantation. Table 3 compares the rates of bridge to urgent heart transplantation or to left ventricular assist device among 
TABLE 3. Rates of bridge to urgent heart transplantation or to ventricular assist device implantation, for patients receiving E-CPR versus veno-arterial ECLS during the same period under noncardiac arrest circumstances

\begin{tabular}{|c|c|c|}
\hline Characteristic & $\begin{array}{c}\text { E-CPR } \\
\text { patients } \\
\end{array}$ & $\begin{array}{c}\text { ECLS patients; } \\
\text { no cardiac arrest } \\
\text { at implantation } \\
\end{array}$ \\
\hline \multicolumn{3}{|l|}{ Overall series } \\
\hline Urgent heart transplantation & 4.1 & 4.8 \\
\hline VAD & 4.1 & 1.9 \\
\hline Any & 8.2 & 6.7 \\
\hline \multicolumn{3}{|c|}{ Survivors at ECLS explantation only } \\
\hline Urgent heart transplantation & 9.5 & 9.8 \\
\hline VAD & 9.5 & 3.8 \\
\hline Any & 19.4 & 13.6 \\
\hline Age $(y)$, mean \pm SD & $47.6 \pm 16^{*}$ & $55.5 \pm 13.9^{*}$ \\
\hline
\end{tabular}

patients who received E-CPR, versus the remaining patients who received veno-arterial ECLS in our institution during the same time period.

\section{Late Results}

The follow-up was $94.5 \%$ complete (1 patient died); average follow-up duration was $15.6 \pm 19.2$ months. Among the 18 patients who left the hospital alive, all were still alive at the latest available follow-up. The Kaplan-Meier survival curve (Figure 2) consequently indicates stable survival after the 16th day after implantation (time point of the last observed death).

Measures of health-related quality of life, as obtained by the SF-36 questionnaire among the survivors at follow-up, are summarized in Table 4. Our patients showed the best

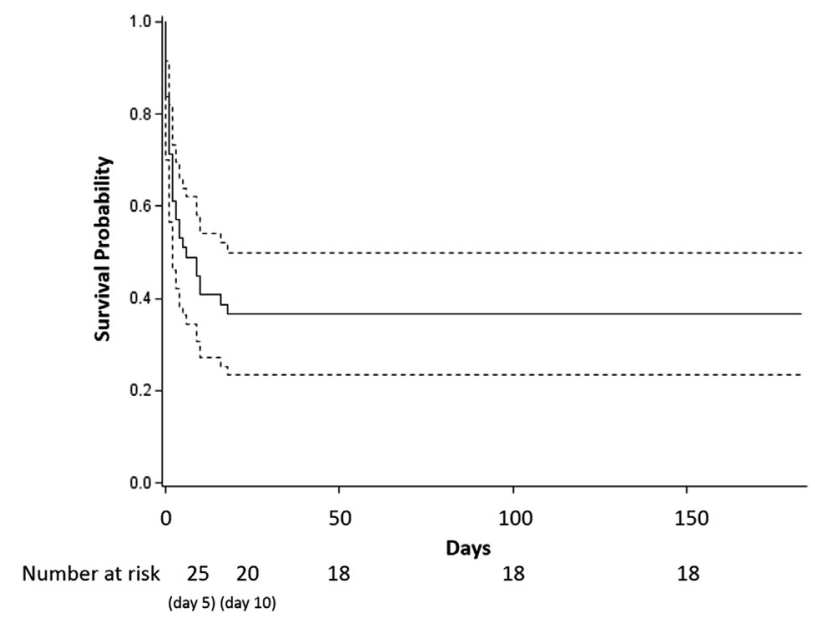

FIGURE 2. Actuarial (Kaplan-Meier) survival curve for patients receiving E-CPR for refractory cardiogenic shock; in-hospital mortality is included. The dashed lines indicate the confidence limits for the estimate.
TABLE 4. Results of quality-of-life scores assessment among survivors at the average 15.6 months follow-up

\begin{tabular}{lc}
\hline \multicolumn{1}{c}{ Score domain } & Mean \pm SD \\
\hline Physical Activity & $70.8 \pm 27.4$ \\
Role Limitation & $63.6 \pm 23.2$ \\
Physical Pain & $81.3 \pm 23.6$ \\
General Health & $62.7 \pm 16$ \\
Vitality & $56.5 \pm 18.8$ \\
Social Activity & $74 \pm 23$ \\
Mental Health & $71.4 \pm 17$ \\
Physical Component Summary & $45.2 \pm 6.8$ \\
Mental Component Summary & $48.3 \pm 7.7$ \\
\hline
\end{tabular}

Best possible score $=100 . S D$, Standard deviation.

scores in the Physical Pain, Social Activity, Mental Health, and Physical Activity domains, whereas the average performance was worse in the Vitality domain. Figure 3 offers a comparison of the average SF-36 scores obtained in our series, versus those previously published for survivors of acute respiratory distress syndrome, through venovenous ECMO, and survivors of postcardiotomy myocardial failure through veno-arterial ECLS. Control

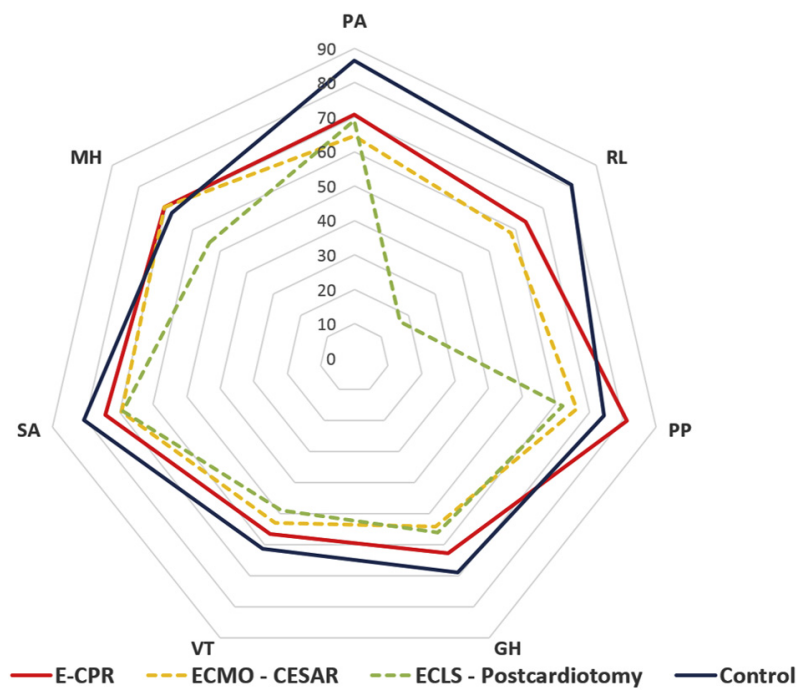

FIGURE 3. Comparison of SF-36 scores observed among patients enrolled in our study (solid red line; E-CPR) with the corresponding SF-36 scores obtained from: patients who survived acute respiratory distress syndrome, through venovenous ECMO (CESAR study, ECMO cohort [14]; yellow dashed line; ECMO-CESAR); patients who survived postcardiotomy myocardial failure through veno-arterial ECLS ([13]; green dashed line; ECLS - postcardiotomy). Comparison with normal age- and gender-matched French population is shown (solid blue line; Control). PA, Physical Activity; $M H$, Mental Health; $R L$, Role Limitation; $S A$, Social Activity; PP, Physical Pain; VT, Vitality; $G H$, General Health; $E$-CPR, extracorporeal cardiopulmonary resuscitation; ECMO - CESAR, extracorporeal membrane oxygenation - conventional ventilatory support versus extracorporeal membrane oxygenation for severe adult respiratory failure; $E C L S$, extracorporeal life support. 
data are for a French age- and gender-matched population without adverse health conditions. ${ }^{10}$ Globally, our patients showed lower average SF-36 scores than did control patients, but in addition, they had better performance than the comparator ECMO/ECLS cohorts.

\section{DISCUSSION}

Since the earliest experiences with E-CPR as a means to help rescue patients from refractory cardiac arrest, 30-day survival in this complex patient subgroup has increased from $24 \%$ then, ${ }^{12,19}$ to approximately $34 \%$ recently, ${ }^{20}$ and to $39 \%$ in selected populations (myocardial infarction-related cardiac arrest $\left.{ }^{1}\right)$. This general trend is confirmed herein. We observed $42.9 \%$ survival at ECLS explantation, and $36.7 \%$ survival at both 30 days and hospital discharge. Our series should be compared with previously published experiences that included only patients who received ECLS during ongoing CPR. Otherwise, the interpretation of previously reported ${ }^{5}$ survival rates of up to $50 \%$ may be misleading. Yet, ECLS guarantees a survival advantage, compared with conventional CPR, if the need continues beyond 10 minutes. ${ }^{21}$ This improvement can be ascribed to better selection of candidates and the increasing experience of E-CPR teams. Prompt decision making and effective organization are crucial for the survival of these critically ill patients. In fact, anoxic brain death of patients treated with E-CPR seems to be 1 major threat in published reports. $^{6,22,23}$

Causes of death on ECLS support are closely related to the major decision-making issues encountered in these patients. Despite the relatively low rate of out-of-hospital cardiac arrest in our series $(12 \%)$, the rate of brain death among E-CPR recipients remains remarkable $(24.5 \%$ overall). Conversely, multiorgan failure is the most prevalent cause of death in our population: end-organ damage induced by cardiogenic shock can be reasonably considered to be the triggering factor for this ominous condition. This concept highlights the importance of prompt and effective CPR, and institution of E-CPR; and in fact, in our study, survivors showed a trend toward significantly shorter average low-flow time, and additionally, absence of no-flow time. Nonetheless, ECLS itself represents a trigger for systemic inflammatory reaction: it has been associated with a "cytokine storm" (tumor necrosis factor- $\alpha$, interleukin 8 , and interleukin6 ), which may propagate organ injury. ${ }^{23}$

Evidence from the animal model suggests that such a mechanism may additionally play a role in delayed pulmonary recovery during venovenous ECLS for severe respiratory failure. ${ }^{24}$ Myocardial failure itself is the less-common determinant of the final outcome once ECLS has been instituted. Fatalities due to failed electrical recovery from cardiac arrest occurred in 2 cases in our series, despite effective ventricular unloading. Early termination of support was the decision made in these cases. Patients who present failed recovery of myocardial contractility during the later days may be bridged to urgent heart transplantation, or to a destination left ventricular assist device ( 2 cases each in our series, $9.5 \%$ of the survivors at the 30th day). However, a reasonable approach to use of transplantation and ventricular assist device resources is limited to patients with survivable levels of end-organ dysfunction.

The proposal has been made that recovery from the initial shock-related injury should be expected during the time period from the 2nd to 5th postimplantation day; ECLS-related end-organ injury would become clinically significant in the later days, and render end-organ recovery unlikely. ${ }^{25}$ Nonsurviving patients presented higher simplified acute physiology scores, lower body temperatures, and higher serum lactate levels at implantation, compared with survivors. In addition, we observed a trend toward longer low-flow time among nonsurvivors. Lower blood $\mathrm{pH}$ was observed among nonsurvivors, although significance was not attained. These data should be interpreted cautiously, because multivariate analysis could not be performed. Nonetheless, these observations suggest the importance of the degree of end-organ injury in determining survival, and may help in the decision-making. Controversy has arisen regarding whether E-CPR should be used outside the referral institution for out-of-hospital cardiac arrest. ${ }^{1,26,27}$ This controversy involves not only clinical factors, but also organizational, public healthcare, economic, and ethical issues. The possibility that sudden cardiac arrest can occur anywhere in the territory, and the prognostic importance of prompt initiation of support when indicated, suggests that ECLS equipment and techniques should be available in all peripheral hospitals that have an intensive care unit. This model would better guarantee that members of all communities have an equal right to access to lifesaving care, even if cardiac arrest occurs far from a tertiary referral center.

On the other hand, the need for surgical and intensive-care expertise, the non-negligible learning curve, and the major social costs support the idea of reserving this technique to tertiary centers. The concept of a territorial network (several peripheral hospitals depending on a single tertiary center) is part of this debate. Given that ECLS is at the frontier in the intersection of cardiology, cardiac surgery, and intensive-care medicine, professionals involved in all these specialties are expected to contribute and develop shared recommendations.

From our experience, we noted that the rate of bridge to heart transplantation is comparable among patients receiving E-CPR, versus the remainder of veno-arterial ECLS cases at our institution (Table 3). The rate of 
long-term ventricular assist device implantation was even higher among refractory cardiac arrest patients. Conclusions from these data are limited by the small sample size; interpretations should take into account the heterogeneous composition of the non-cardiac arrest population (including patients receiving ECLS for early graft failure and postcardiotomy myocardial failure) and the lower average age among the E-CPR patients. Nevertheless, this information suggests that urgent transplantation and long-term ventricular assist device use are as effective among rescued cardiac arrest patients as they are in the general ECLS population. Therefore, the E-CPR patients should be submitted to the same decisional criteria as conventional ECLS patients, although particular attention must be devoted to the assessment of brain death and nonsurvivable end-organ injury.

One major finding of the present work is the demonstration of stable survival at the midterm follow-up among patients who were discharged from the hospital. Concerning the assessment of quality of life, higher scores were observed for the Mental Health and related domains; lower scores were observed for the General Health and Vitality domains. This finding may be interpreted as a consequence of the early to midterm follow-up of long-term hospitalization and intensive-care unit stay. The subtending disease, intensive-care unit-related neuropathy, hyponutrition, and the high rate of complications may explain this finding. Improvements in these domains and more-complete recovery at later intervals can be reasonably expected.

Survivors of cardiac arrest for whom E-CPR was used showed herein worse health-related quality-of-life scores than the healthy matched French population, as the expression of severe disease and prolonged hospitalization. Our E-CPR patients presented better average scores in all SF-36 domains, compared with those from previous studies of ECLS patients, ${ }^{13}$ venovenous ECMO patients, ${ }^{14}$ and survivors of postcardiotomy myocardial failure through ECLS $^{13}$ (Figure 3). This finding may be attributed to various characteristics of the comparator populations. Namely, patients on venovenous ECMO are especially likely to experience particularly long and debilitating hospitalization in a critical care environment. The patients enrolled in the study by Wang and colleagues ${ }^{13}$ all presented postcardiotomy myocardial failure and structural heart disease, with inherent negative prognostic significance.

Collectively, our data suggest that patients who survive acute events as a result of E-CPR may attain satisfactory health-related quality of life compared with similar populations of critical care patients. Similarly, the SF-36 Mental and Physical summary scores were comparable to those obtained among survivors of cardiac arrest who did not receive E-CPR treatment. ${ }^{18}$ The present paper reports, for the first time, quality-of-life measures among patients who survive refractory cardiac arrest as a result of E-CPR. Therefore, the practice of E-CPR is justified by the possibility of achieving good functional results in survivors.

The present investigation is limited by the use of retrospective analysis of data; this limitation is partially offset by the prospective inclusion of patients' information in a dedicated database. Another limitation is the lack of a control group (survivors of cardiac arrest who did not receive E-CPR). On the other hand, we limited inclusion to patients who received E-CPR during ongoing external cardiac massage, thereby focusing on a more-select population than previous reports. ${ }^{5}$ A further minor limitation is that the rate of thromboembolic and hemorrhagic complications is given in aggregate form.

\section{CONCLUSIONS}

Use of E-CPR is an effective strategy that allows for saving lives in a population of severely compromised patients. This domain is probably the most challenging one within an ECLS program. ${ }^{28}$ The survival at ECLS explantation reported herein is among the highest published so far. Patients who survive the initial cardiac arrest injury, owing to E-CPR, may subsequently enter the same decision-making algorithm as patients who received ECLS during non-cardiac arrest circumstances. Nonetheless, high rates of brain death and nonsurvivable end-organ injury are common among patients rescued from cardiac arrest, and may preclude bridging to urgent transplantation or a ventricular assist device in a significant proportion of cases.

Overall, about one third of patients in our experience survived at both discharge and midterm follow-up. Remarkably, survival tends to remain stable after discharge. Health-related quality of life among survivors is better than or comparable to that in previous investigations of ECLS and/or ECMO recipients, or cardiac arrest survivors. These findings support the practice of E-CPR, provided that adequate patient selection is performed, to avoid unjustified use of hospital resources.

\section{Conflict of Interest Statement}

Authors have nothing to disclose with regard to commercial support.

The authors are grateful to Pascale Roualt, Veronique Desriac, and Anne Ingels for their invaluable contributions to data collection and analysis.

\section{References}

1. Demondion P, Fournel L, Golmard JL, Niculescu M, Pavie A, Leprince P. Predictors of 30-day mortality and outcome in cases of myocardial infarction with cardiogenic shock treated by extracorporeal life support. Eur J Cardiothorac Surg. 2014;45:47-54. 
2. Chen Y, Chao A, Yu H, Ko WG, Wu IH, Chen R, et al. Analysis and results of prolonged resuscitation in cardiac arrest patients rescued by extracorporeal membrane oxygenation. J Am Coll Cardiol. 2003;41:197-203.

3. Ruttmann E, Weissenbacher A, Ulmer H, Muller H, Hofer D, Kilo J, et al. Prolonged extracorporeal membrane oxygenation-assisted support provides improved survival in hypothermic patients with cardiocirculatory arrest. $J$ Thorac Cardiovasc Surg. 2007; 134:594-600.

4. Megarbane B, Leprince P, Deye N, Resiere D, Guerrier G, Rettab S, et al. Emergency feasibility in medical intensive care unit of extracorporeal life support for refractory cardiac arrest. Intensive Care Med. 2007;33:758-64.

5. Chen J, Ko W, Yu H, Lai L, Huang S, Chi N, et al. Analysis of the outcome for patients experiencing myocardial infarction and cardiopulmonary resuscitation refractory to conventional therapies necessitating extracorporeal life support rescue. Crit Care Med. 2006;34:950-7.

6. Thiagarajan R, Brogan $T$, Scheurer M, Laussen P, Rycus P, Bratton S. Extracorporeal membrane oxygenation to support cardiopulmonary resuscitation in adults. Ann Thorac Surg. 2009;87:778-85.

7. Combes A, Leprince P, Luyt CE, Trouillet JL, Chastre J. Extracorporeal membrane oxygenation (ECMO) for cardiopulmonary support (Assistance cardiorespiratoire par extracorporeal membrane oxygenation (ECMO). Réanimation. 2009;18:420-7.

8. Beckmann A, Benk C, Beyersdorf F, Haimerl G, Merkle F, Mestres C, et al; ECLS working group. Position article for the use of extracorporeal life support in adult patients. Eur J Cardiothorac Surg. 2011;40:676-80.

9. Brinkman J, Bakhshi-Raiez F, Abu-Hanna A, de Jonge E, Bosman RJ, Peelen R, et al. External validation of Acute Physiology and Chronic Health Evaluation IV in Dutch intensive care units and comparison with Acute Physiology and Chronic Health Evaluation II and Simplified Acute Physiology Score II. J Crit Care. 2011;26:e11-8.

10. Leplège A, Ecosse E, Verdier A, Perneger T. The French SF-36 Health Survey: translation, cultural adaptation and preliminary psychometric evaluation. J Clin Epidemiol. 1998;51:1013-23.

11. Aaronson NK, Acquadr C, Alonso J, Apolone G, Bucquet D, Bullinger M, et al. International Quality of Life Assessment (IQOLA) Project. Qual Life Res. 1992; $1: 349-51$.

12. Doll N, Kiaii B, Borger M, Bucerius J, Kramer K, Schmitt V, et al. Five-years results of 219 consecutive patients treated with extracorporeal membrane oxygenation for refractory postoperative cardiogenic shock. Ann Thorac Surg. 2004;77:151-7.

13. Wang J, Han J, Jia Y, Zeng W, Shi J, Hou X, et al. Early and intermediate results of rescue extracorporeal membrane oxygenation in adult cardiogenic shock. Ann Thorac Surg. 2009;88:1897-903.

14. Peek GJ, Mugford M, Tiruvoipati R, Wilson A, Allen E, Thalanany MM, et al. Efficacy and economic assessment of conventional ventilatory support versus extracorporeal membrane oxygenation for severe adult respiratory failure (CESAR): a multicentre randomised controlled trial. Lancet. 2009;17(374): 1351-63.

15. Kugler C, Malehsa D, Tegtbur U, Guetzlaff E, Meyer A, Bara C, et al. Health-related quality of life and exercise tolerance in recipients of heart transplants and left ventricular assist devices: a prospective, comparative study. J Heart Lung Transplant. 2011;30:204-10.

16. Freitas P, Haida A, Bousquet M, Richard L, Mauriège P, Giraud T. Short-term impact of a 4-week intensive cardiac rehabilitation program on quality of life and anxiety-depression. Ann Phys Rehabil Med. 2011;54:132-3.

17. MacIver J, Ross H. Quality of life and left ventricular assist device support. Circulation. 2012;126:866-74.

18. Graf J, Muhlhoff C, Doig S, Reinartz S, Bode K, Dujardin R, et al. Health care costs, long-term survival, and quality of life following intensive care unit admission after cardiac arrest. Crit Care. 2008;12:R92.

19. Schwarz B, Mair P, Margreiter J, Pomaroli A, Hoerman C, Bonatti J, et al. Experience with percutaneous venoarterial cardiopulmonary bypass for emergency circulatory support. Crit Care Med. 2003;31:758-64.

20. Haneya A, Philipp A, Diez C, Schopka S, Bein T, Zimmermann M, et al. A 5-year experience with cardiopulmonary resuscitation using extracorporeal life support in non-postcardiotomy patients with cardiac arrest. Resuscitation. 2012;83: 1331-7.

21. Chen Y, Lin J, Yu H, Ko W, Jerng JS, Chang WT, et al. Cardiopulmonary resuscitation with assisted extracorporeal life-support versus conventional cardiopulmonary resuscitation in adults with in-hospital cardiac arrest: an observational study and propensity analysis. Lancet. 2008;372:554-61.

22. Massetti M, Tasle M, Le Page O, Dereck R, Babatasi G, Buklas D, et al. Back from irreversibility: extracorporeal life support for prolonged cardiac arrest. Ann Thorac Surg. 2005;79:178-84.

23. Risnes I, Wagner K, Ueland T, Mollnes T, Aukrust P, Svennevig J. Interleukin-6 may predict survival in extracorporeal membrane oxygenation treatment. Perfusion. 2008;23:173-8.

24. Shi J, Chen Q, Yu W, Shen J, Gong C, He J, et al. Continuous renal replacement therapy reduces the systemic and pulmonary inflammation induced by venovenous extracorporeal membrane oxygenation in a porcine model. Artif Organs. 2014;38:215-23.

25. Pagani FD, Aaronson KD, Dyke DB, Wright S, Swaniker F, Bartlett RH. Assessment of an extracorporeal life support to LVAD bridge to heart transplant strategy. Ann Thorac Surg. 2000;70:1977-84.

26. Kagawa E, Inoue I, Kawagoe T, Ishihara M, Shimatani Y, Kurisu S, et al. Assessment of outcomes and differences between in- and out-of-hospital cardiac arrest patients treated with cardiopulmonary resuscitation using extracorporeal life support. Resuscitation. 2010;81:968-73.

27. Le Guen M, Nicolas-Robin A, Carreira S, Raux M, Leprince P, Riou B, et al. Extracorporeal life support following out-of-hospital refractory cardiac arrest. Crit Care. 2011;15:R29.

28. Flécher E, Anselmi A, Corbineau H, Langanay T, Verhoye JP, Félix C, et al. Current aspects of extracorporeal membrane oxygenation in a tertiary referral centre: determinants of survival at follow-up. Eur J Cardio-Thorac Surg. 2014;46:665-71.

Key Words: extracorporeal membrane oxygenation, resuscitation, outcomes, health policy 